\title{
Конденсация экситонных поляритонов в микрорезонаторах, индуцированная магнитным полем
}

\author{
() В.П. Кочерешко*+, Д.В. Авдошина*, P. Savvidis ${ }^{\bullet}$, S.I. Tsintzos ${ }^{\bullet}$, Z. Hatzopoulos ${ }^{\bullet}$, \\ A.V. Kavokin ${ }^{+\diamond}$, L. Besombes ${ }^{\square}$, H. Mariette $\square$ \\ * Физико-технический институт им. А.Ф. Иоффе Российской академии наук, \\ 194021 Санкт-Петербург, Россия \\ + Санкт Петербургский государственный университет, \\ 199034 Санкт-Петербург, Россия \\ - Department of Materials Science \& Technology, \\ University of Crete, Greece \\ $\checkmark$ School of Physics and Astronomy, University of Southampton, \\ Southampton, SO171BJ, UK \\ $\square$ Institut Neel, CNRS, \\ Grenoble, France \\ E-mail: Vladimir.Kochereshko@mail.ioffe.ru
}

(Получена 27 апреля 2016 г. Принята к печати 10 мая 2016 г.)

Исследованы спектры фотолюминесценции экситонных поляритонов в микрорезонаторах в условиях 3-мерного квантования в зависимости от плотности оптического возбуждения и магнитного поля. По поведению степени круговой поляризации экситонной люминесценции в магнитном поле установлено, что при повышении концентрации экситонов они конденсируются на самом нижнем по энергии зеемановском подуровне.

\section{1. Введение}

Исследование поляритонов (смешанных экситон фотонных состояний) в микрорезонаторах в последние годы привлекает пристальное внимание исследователей в связи с перспективой наблюдения бозе-конденсации поляритонов в такой системе. Конденсат из бозе-частиц дает нам пример нового, необычного состояния вещества — квантовых жидкостей. Они обладают многими непривычными свойствами, такими как сверхтекучесть, образование квантовых вихрей, макроскопическая когерентность движения частиц и т.д. К сожалению, практически единственный пример бозе-жидкости, встречающийся в природе, это жидкий гелий. Долгое время стояла задача найти и другие примеры бозе-конденсированного состояния вещества. Это удалось осуществить для газа атомов рубидия, и за наблюдение бозе-конденсации была присвоена Нобелевская премия в 2001 году [1]. Однако бозе-конденсацию в этой системе удалось наблюдать только в „экстремальных условиях“, при предельно низких температурах, в магнитных ловушках.

Другим хорошим кандидатом для наблюдения бозеконденсации является система экситонов в полупроводнике. Экситоны являются бозе-частицами, их эффективная масса мала и это позволило бы наблюдать конденсацию экситонов при разумных температурах. Еще легче наблюдать явление бозе-конденсации для экситонных поляритонов в микрорезонаторах. Их масса на несколько порядков меньше массы экситона и для них можно было бы наблюдать явление бозе-конденсации даже при комнатной температуре. При этом экситонные поляритоны являются композитными бозонами, состоящими из двух фермионов. Эта их особенность позволяет проследить переход от бозе-системы к ферми-системе [2], что представляет значительный интерес для фундаментальной физики. Дело, однако, осложняется тем, что поляритоны живут довольно короткое время и за время жизни могут не успеть термализоваться и сконденсироваться. Эту трудность преодолеть нельзя и приходится довольствоваться неравновесной конденсацией [3]. Тем не менее поляритоны в микрорезонаторах в настоящее время являются главным объектом исследований бозе-систем.

В данной работе исследовалась зависимость спектров поляризованной фотолюминесценции экситонных поляритонов в микрорезонаторах с 3-мерным квантованием поляритонов в зависимости от внешнего магнитного поля и от интенсивности оптического возбуждения при температуре $5 \mathrm{~K}$.

\section{2. Эксперимент}

Исследуемые структуры представляли собой мезы, изготовленные из микрорезонаторов. Микрорезонатор

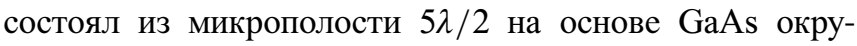
женной двумя брэгговскими зеркалами, состоящими из 32 слоев $\mathrm{AlAs} / \mathrm{Al}_{0.15} \mathrm{Ga}_{0.85} \mathrm{As}$. В микрополость помещались четыре квантовые ямы, расположенные в пучностях электромагнитного поля. На основе таких микрорезонаторов был изготовлен набор мез диаметром от 1 до 40 микрон. Добротность микрорезонатора достигала 20000. В микрорезонаторах имел место режим сильной экситон-фотонной связи с расщеплением Раби порядка 10 мэВ. Спектры фотолюминесценции возбуждались импульсами Ti:Sa-лазера с длительностью импульса 2 пикосекунды и частотой повторения 10 Кгц с 


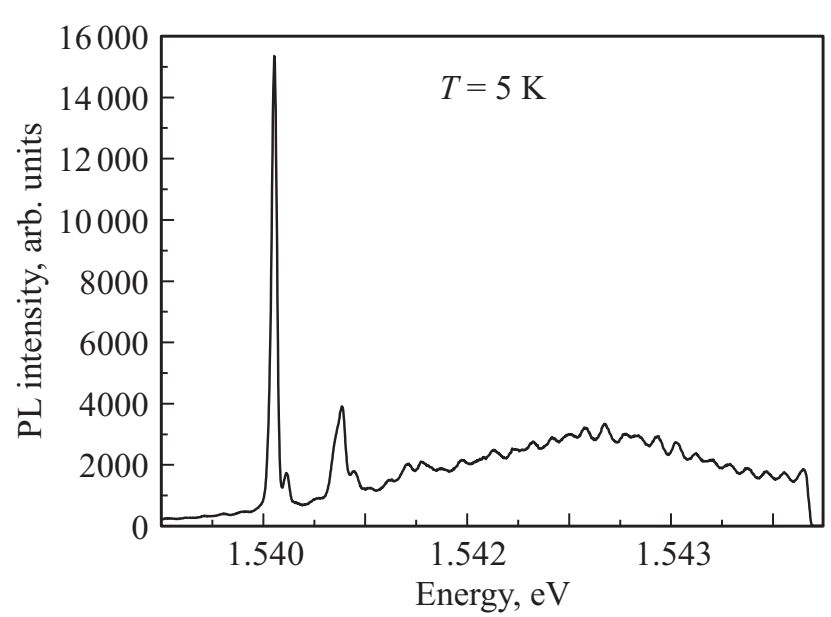

Рис. 1. Спектр фотолюминесценции, снятый от мезы диаметром 5 мкм при температуре $5 \mathrm{~K}$, при интенсивности оптического возбуждения, близкой к порогу возникновения узких линий латерального квантования поляритонов в спектре.

энергией 1.62 эВ, что соответствует, с одной стороны, области прозрачности брэгговских зеркал, а с другой области прозрачности барьерных слоев AlGaAs.

При малой интенсивности возбуждения спектр фотолюминесценции мез представлял собой широкую полосу излучения, простирающуюся от примерно 1.540 до 1.550 эВ практически независимо от диаметра мезы. При превышении интенсивности возбуждения выше некоторого порогового значения на длинноволновом крыле этой полосы появляется один узкий пик полушириной менее $1 / 2$ мэВ (рис. 1). Интенсивность этого пика сначала очень быстро нарастает с ростом интенсивности возбуждения, а затем насыщается.

Большая ширина полосы излучения при малой интенсивности возбуждения связана с потерями через боковую поверхность мез. Когда интенсивность возбуждения превышает эти потери, в спектре начинают проявляться состояния латерального квантования экситонов.

При дальнейшем возрастании интенсивности возбуждения коротковолновее этого пика последовательно появляются другие узкие пики. Их число и энергетическое расстояние между ними зависит от латерального размера мезы. Так, в мезах диаметром 40 мкм наблюдалась всего одна линия, а в мезах диаметром, например, 10 мкм наблюдалось 3 линии. В мезе диаметром 5 мкм наблюдалось до 5 линий латерального квантования поляритонов (смешанных состояний экситона и фотона) на энергиях. Эти пики, очевидно, связаны с латеральным квантованием экситонных поляритонов в условиях нелинейного режима, который соответствует поляритонной лазерной генерации (когда генерация происходит на нижней поляритонной ветви). Экситонная природа узких линий фотолюминесценции подтверждается тем, что они испытывают заметный диамагнитный сдвиг и зеемановское расщепление в магнитном поле. Было обнаружено также, что с увеличением магнитного поля пороговое значение интенсивности возбуждения для появления узких линий в спектре уменьшилось в десятки раз.

\section{3. Результаты}

В магнитных полях наблюдался диамагнитный сдвиг всех линий, связанных с латеральным квантованием экситонных поляритонов в сторону больших энергий, и их зеемановское расщепление. Экситонный $g$-фактор, связанный с зеемановским расщеплением, оказался равным $g_{\text {exc }}=0.24$, а диамагнитный сдвиг составил 2.9 мэВ в поле $11 \mathrm{~T}$. Полученные величины типичны для квантовой ямы на основе GaAs шириной порядка $60 \AA$, что подтверждает экситонную природу этих линий.

В мезах, в которых была достаточно малая расстройка между фотонной и экситонной модами, были обнаружены следующие явления. При фиксированном магнитном поле, при слабом уровне оптического возбуждения ниже некоторого порога, соотношение интенсивностей верхней и нижней зеемановских компонент нижнего по энергии уровня латерального квантования экситона составляло примерно $1: 2$, что соответствует степени поляризации $30 \%$.

Естественно предположить, что благодаря сильной связи с электромагнитным полем, время жизни экситона в микрорезонаторе много короче времени его спиновой релаксации. Тогда эта величина соответствует больцмановскому распределению экситонов на зеемановских подуровнях при температуре $5 \mathrm{~K}$ (рис. 2,a).

При повышении интенсивности оптического возбуждения вблизи порога при фиксированном магнитном поле происходило резкое перераспределение соотношения интенсивностей этих зеемановских компонент. Интенсивность нижнего зеемановского подуровня возрастала пороговым образом (рис. 2,b). При больших интенсивностях возбуждения в спектре оставалась только одна линия соответствующая нижнему зеемановскому подуровню (рис. 2,c). При дальнейшем увеличении интенсивности возбуждения наблюдалось уширение этой линии и ее коротковолновый сдвиг.

В зависимости от магнитного поля при фиксированной интенсивности возбуждения наблюдалось похожее перераспределение интенсивностей экситонной люминесценции. В малых магнитных полях наблюдалось обычное больцмановское распределение интенсивностей (рис. 3,a), а в больших полях в спектре оставалась только одна линия, соответствующая нижнему зеемановскому подуровню (рис. $3, b)$.

\section{4. Обсуждение}

Естественно считать, что интенсивность люминесценции наблюдаемых линий пропорциональна заселенности соответствующих энергетических уровней. Тогда наши результаты указывают на то, что при увеличении концентрации экситонов при фиксированном магнитном поле 

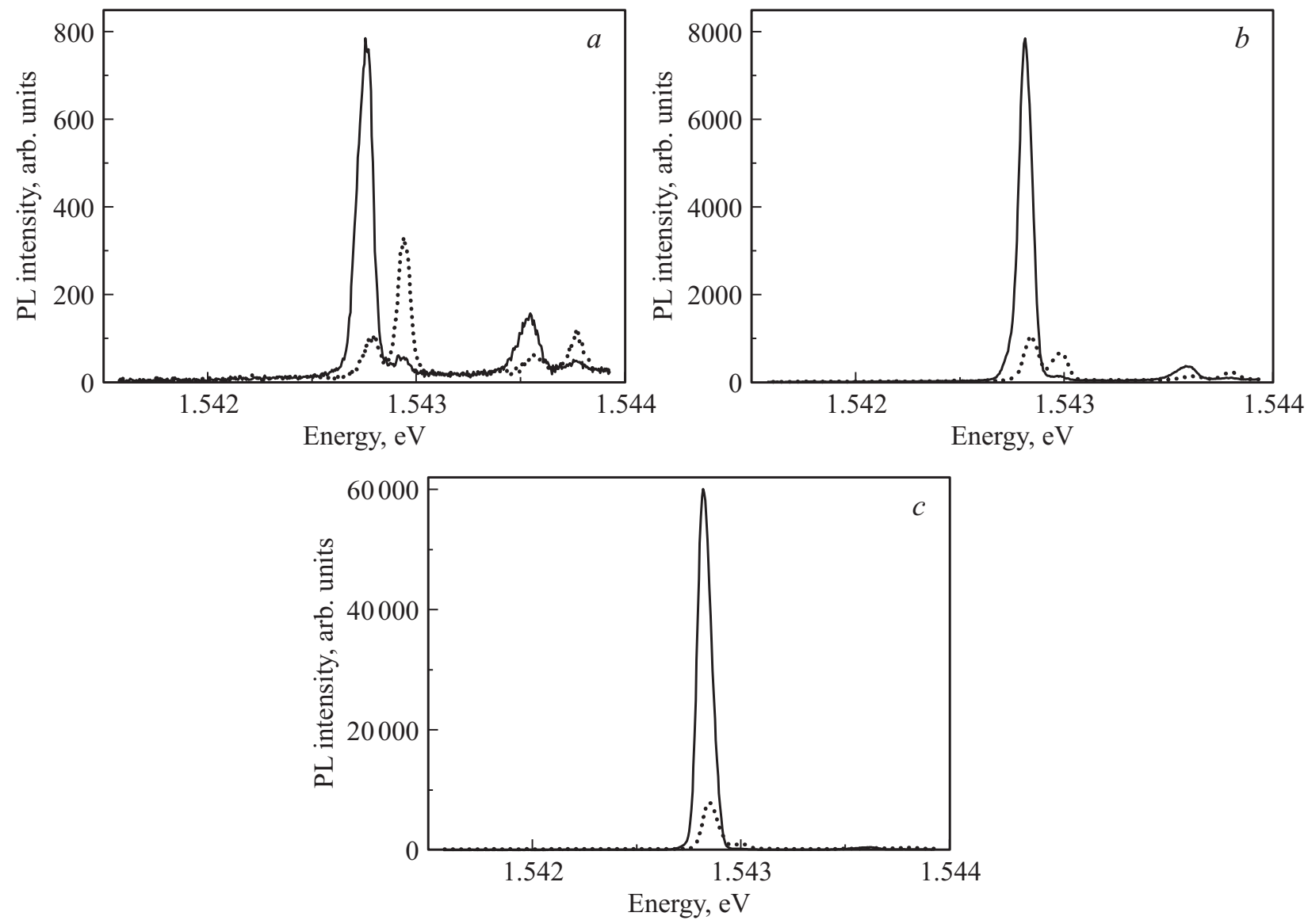

Рис. 2. Зависимость спектров фотолюминесценции экситонных поляритонов от интенсивности оптического возбуждения при фиксированной величине магнитного поля $11 \mathrm{~T}$, снятых в двух круговых поляризациях $\sigma^{+}$(сплошная кривая) и $\sigma^{-}$(пунктир): $a-$ при интенсивности возбуждения ниже порога; $b-$ при интенсивности возбуждения вблизи порога; $c-$ при интенсивности возбуждения выше порога.
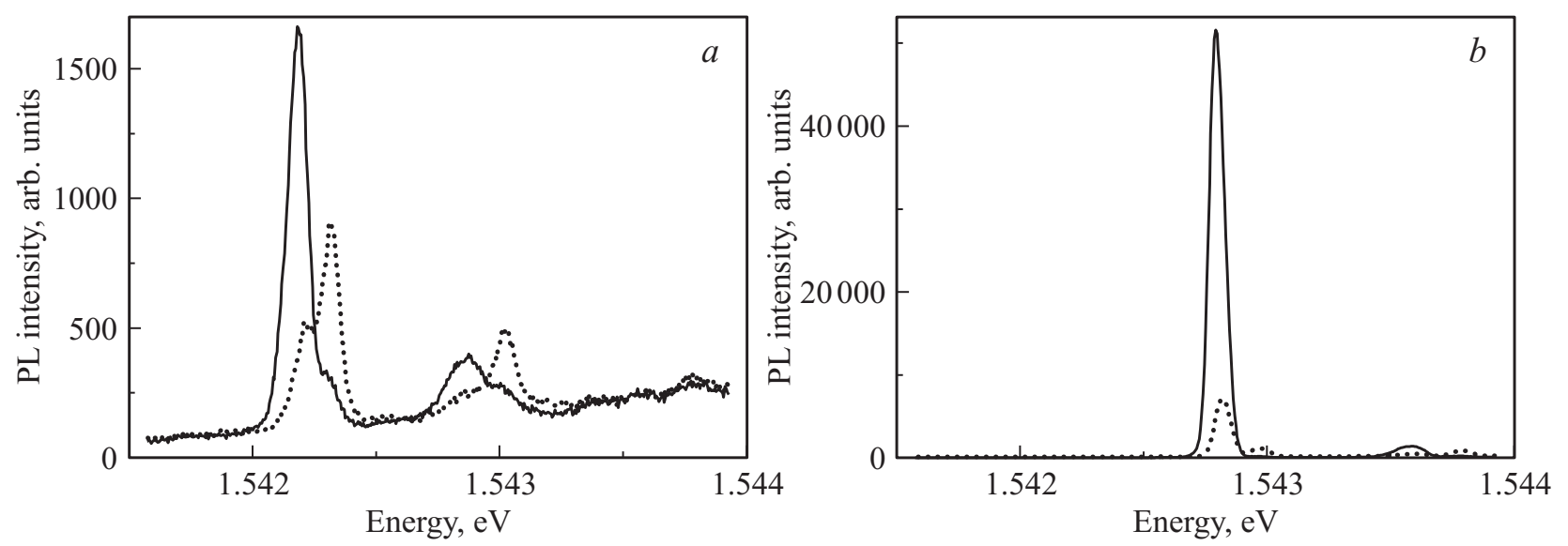

Рис. 3. Зависимость спектров фотолюминесценции экситонных поляритонов от величины магнитного поля при фиксированной интенсивности оптического возбуждения, снятых в двух круговых поляризациях $\sigma^{+}$(сплошная кривая) и $\sigma^{-}$(пунктир): $a-$ при величине магнитного поля $8 \mathrm{~T} ; b-$ при величине магнитного поля $11 \mathrm{~T}$.

или при увеличении магнитного поля при фиксированной концентрации экситонов экситонам оказывается выгодным собраться на самом нижнем энергетическом уровне.
Статистика Бозе-Эйнштейна, которой подчиняются экситоны, позволяет любому числу экситонов заселять один энергетический уровень. Однако при конечной температуре должны быть заселены и более высокие 


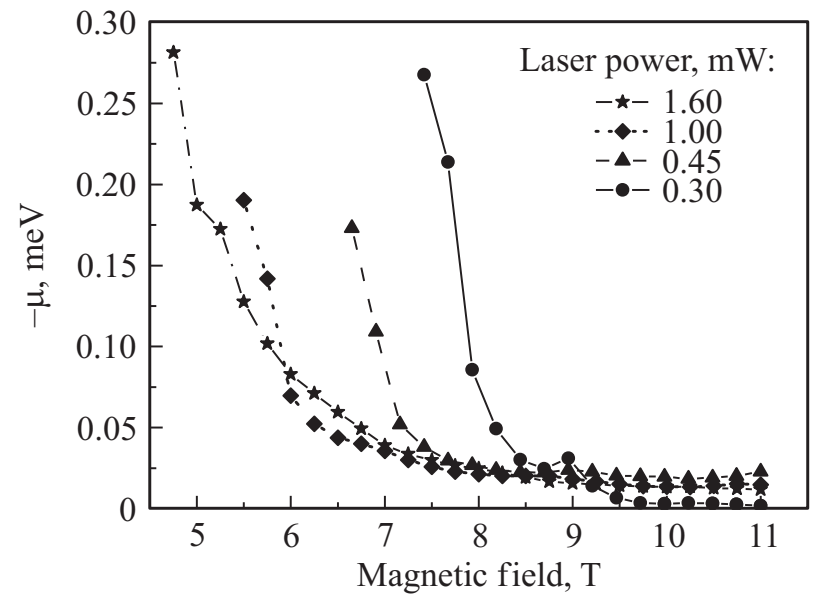

Рис. 4. Зависимость величины химического потенциала ансамбля экситонных поляритонов от напряженности магнитного поля при разных уровнях оптического возбуждения.

по энергии уровни. В нашем случае зеемановское расщепление составляет всего 0.15 мэВ, что даже меньше $k T=0.5$ мэВ, и, следовательно, верхний зеемановский подуровень должен быть в достаточной степени заселен. Однако при большой концентрации экситонов создаваемой оптическим возбуждением, оказалось, что все экситоны (или почти все) собираются на самом нижнем энергетическом уровне. Это возможно для статистики Бозе-Эйнштейна, если химический потенциал ансамбля экситонов оказался близок к нулю.

На рис. 4 представлена зависимость химического потенциала ансамбля экситонных поляритонов от магнитного поля при разных уровнях оптического возбуждения. Величина химического потенциала определялась из отношения интенсивностей зеемановских компонент. Естественно предположить, что отношение интенсивностей этих компонент пропорционально отношению заселенностей соответствующих подуровней.

Примем за начало отсчета энергии энергию низшего зеемановского подуровня. В соответствии с распределением Бозе-Эйнштейна при температуре $T$, в состоянии равновесия, на нижнем зеемановском подуровне имеется $n_{0}$ экситонов:

$$
n_{0}=\frac{1}{\exp (-\mu / k T)-1} .
$$

На втором зеемановском подуровне будет $n_{1}$ экситонов:

$$
n_{1}=\frac{1}{\exp ((E-\mu) / k T)-1} .
$$

Здесь $E=\mu_{\mathrm{B}} g H$ - энергия зеемановского расщепления в магнитном поле $H, \mu-$ химический потенциал ансамбля экситонов. Отсюда, зная отношение концентраций экситонов $n_{1} / n_{0}=\rho$ и величину зеемановского расщепления $E$, можем найти $\mu$ :

$$
\mu=k T\{\ln (1-\rho \exp (E / k T))-\ln (1-\rho)\} .
$$

Эта величина как функция магнитного поля и представлена на рис. 4.

Из рисунка видно, что химический потенциал ансамбля экситонных поляритонов стремится к нулю с ростом магнитного поля. Причем чем меньше плотность возбуждения, а следовательно, и плотность ансамбля экситонов, тем больше величина магнитного поля, при котором химический потенциал резко падает.

Такое сильное влияние магнитного поля на экситонную конденсацию, вероятнее всего, связано с тем, что магнитное поле уменьшает радиус экситона и тем самым уменьшает отталкивание между экситонами, а следовательно, и химический потенциал. Еще одна возможность для магнитного поля влиять на конденсацию поляритонов связана с тем, что в наших структурах, благодаря диамагнитному сдвигу экситона в магнитном поле, расстройка между фотонной и экситонной модами уменьшается и увеличивается смешивание экситонов с фотонами. Это приводит к уменьшению эффективной массы поляритона и облегчает конденсацию экситонов.

\section{5. Заключение}

Исследованы спектры фотолюминесценции экситонных поляритонов в микрорезонаторах в условиях 3-мерного квантования в зависимости от плотности оптического возбуждения и магнитного поля. Обнаружено, что при повышении концентрации экситонов в фиксированном магнитном поле или при увеличении магнитного поля при фиксированной концентрации экситонов все экситоны собирались на самом нижнем уровне энергии. Построена зависимость химического потенциала системы экситонов от магнитного поля. Установлено, что химический потенциал системы экситонов обращается в нуль, что отражает явление конденсации экситонов на самом нижнем энергетическом уровне.

Авторы благодарны за частичную финансовую поддержку РФФИ, CNRS и Отделению физических наук PAH.

\section{Список литературы}

[1] Э.А. Корнелл, К.Э. Виман. УФН, 173 (12), 1319 (2003).

[2] V.P. Kochereshko, M.V. Durnev, L. Besombes, H. Mariette, V.F. Sapega, A. Askitopoulos, I.G. Savenko, T.C.H. Liew, I.A. Shelykh, A.V. Platonov, S.I. Tsintzos, Z. Hatzopoulos, P.G. Savvidis, V.K. Kalevich, M.M. Afanasiev, V.A. Lukoshkin, C. Schneider, M. Amthor, C. Metzger, M. Kamp, S. Hoefling, P. Lagoudakis, A. Kavokin. Sci. Rept., 6, 20091 (2016).

[3] A. Imamoglu, R.J. Ram, S. Pau, Y. Yamamoto. Phys. Rev. A, 53, 4250 (1996).

Редактор А.Н. Смирнов 


\section{Condensation of exciton polaritons} in microcavity induced by magnetic fields

V.P. Kochereshko*+, D.V. Avdoshina*, P. Savvidis ${ }^{\bullet}$,

S.I. Tsintzos ${ }^{\bullet}$, Z. Hatzopoulos ${ }^{\bullet}$, A.V. Kavokin ${ }^{+\diamond}$,

L. Besombes ${ }^{\square}, H$. Mariette ${ }^{\square}$

* Ioffe Institute,

194021 St. Petersburg, Russia

+ Saint Petersburg State University,

199034 St. Petersburg, Russia

- Department of Materials Science \& Technology,

University of Crete,

Greece

$\checkmark$ School of Physics and Astronomy,

University of Southampton,

Southampton, SO171BJ, UK

$\square$ Institut Neel, CNRS,

Grenoble, France

Abstract Photoluminescence spectra of exciton polaritons in microcavities in a condition of 3-dimensional quantization were studied as a function of the density of the optical excitation and magnetic fields. The behavior of the degree of circular polarization of the exciton luminescence in a magnetic field shows that when the concentration of excitons reach some threshold they condense on the lowest Zeeman sublevel. 